\title{
Atlantoaxial Synovial Cyst Associated with Instability in a Chihuahua
}

\author{
Franck Forterre, ${ }^{1,2}$ Núria Vizcaino Reves, ${ }^{1,3}$ Christina Stahl,, ${ }^{1,4}$ Stephan Rupp, ${ }^{5}$ \\ and Karine Gendron ${ }^{1,4}$
}

${ }^{1}$ Small Animal Clinic, Department of Clinical Veterinary Medicine, Vetsuisse Faculty, University of Berne, 3012 Berne, Switzerland

${ }^{2}$ Department of Neurosurgery, Vetsuisse Faculty, University of Berne, Länggassstrasse 128, 3012 Berne, Switzerland

${ }^{3}$ Department of Surgery, Vetsuisse Faculty, University of Berne, Länggassstrasse 128, 3012 Berne, Switzerland

${ }^{4}$ Department of Radiology, Vetsuisse Faculty, University of Berne, Länggassstrasse 128, 3012 Berne, Switzerland

${ }^{5}$ Tierklinik Hofheim, Im Langgewann 9, am Taunus, 65719 Hofheim, Germany

Correspondence should be addressed to Franck Forterre, franck.forterre@vetsuisse.unibe.ch

Received 30 December 2011; Accepted 1 February 2012

Academic Editors: J. Lakritz and F. Mutinelli

Copyright ( $) 2012$ Franck Forterre et al. This is an open access article distributed under the Creative Commons Attribution License, which permits unrestricted use, distribution, and reproduction in any medium, provided the original work is properly cited.

\begin{abstract}
Objective. To describe an atlantoaxial degenerative cyst associated with instability. Animal. Chihuahua, male, 5 years old. Methods. Ever since colliding with a large dog two years prior to presentation, the dog suffered recurrent episodes of intractable cervical pain. Over time, the pain attacks increased in frequency and intensity. On presentation, pain was clinically localized to the high cervical region. No neurological deficits were observed. CT and MRI revealed an atlantoaxial degenerative articular cyst associated with instability, causing cervicomedullary compressive myelopathy. On MRI the cyst appeared hypointense in T1W and hyperintense in T2-weighted sequences, with rim enhancement. The dog was treated surgically by cyst fenestration and ventral stabilization using a $1.5 \mathrm{~mm}$ Butterfly Locking plate and cancellous bone graft placed within the atlantoaxial joint after cartilage removal. Histological examination of a sample of the cyst wall confirmed a degenerative articular cyst. The dog recovered uneventfully after surgery and remained pain free throughout the 2-year followup. Conclusion. Atlantoaxial degenerative articular cyst associated with instability is a rare finding in dogs. Clinical Relevance. The presence of an atlantoaxial degenerative articular cyst appears not to worsen the prognosis of instability treatment. Atlantoaxial fusion and cyst fenestration may provide good long-term results.
\end{abstract}

\section{Introduction}

Extradural spinal cysts occurring in dogs are relatively uncommon [1-5]. Canine spinal synovial cysts have been described in the cervical spine of large breed dogs, and at the thoracolumbar and lumbosacral junctions $[1,2,4,6]$.

Cysts associated with the atlantoaxial joint are an infrequent cause of cervicomedullary compression in humans [7] and, to the authors' knowledge, have not previously been described in dogs. The goal of the present short communication is to present a case of atlantoaxial cyst associated with instability in a dog.

\section{Case History}

2.1. History. A 5-year-old male Chihuahua was referred to our clinic with a suspicion of atlantoaxial instability because of recurrent cervical pain. Clinical signs had developed two years prior following a collision with a large dog. Episodes of pain had become more frequent and severe in the few months preceding the dog's presentation. No improvement was observed after an oral steroid therapy instituted by the private veterinarian.

2.2. Clinical Examination. The dog presented with a low head carriage. Physical examination, including a complete neurological examination, was otherwise normal. Pain could only be demonstrated upon gentle palpation of the high cervical region. Because of the suspected diagnosis, excessive manipulation of the neck was avoided. Based on the clinical findings, the lesion was localized in the upper cervical region. Differential diagnoses included atlantoaxial instability, cervical fracture/luxation, intervertebral disc disease, neoplasia, 


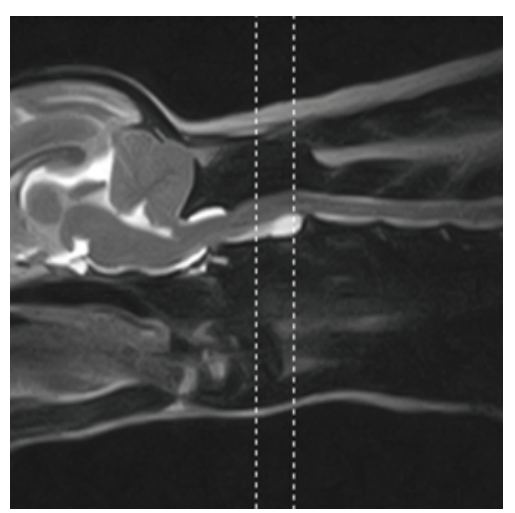

(a)

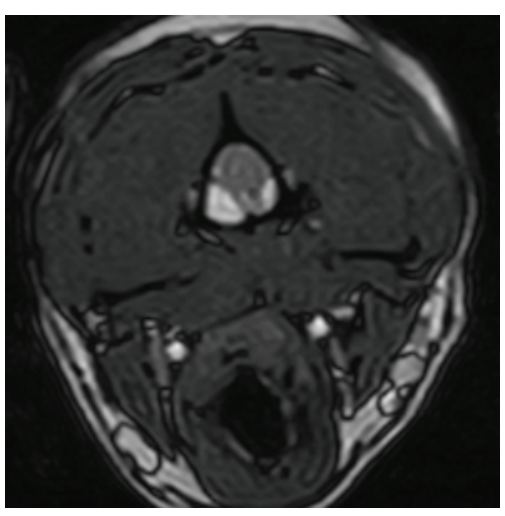

(b)

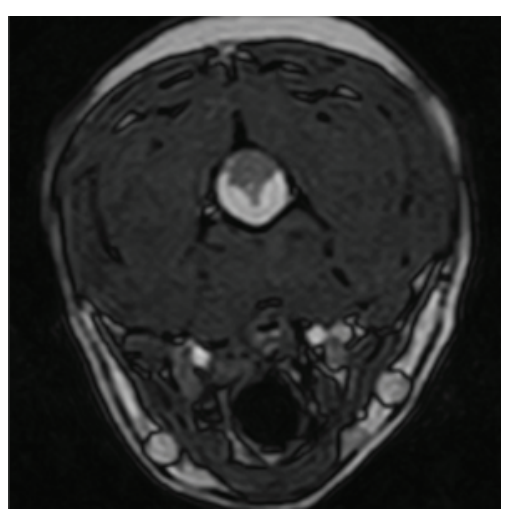

(c)

FIGURE 1: (a) Sagittal T2-weighted MR image showing a hyperintense cyst compressing the spinal cord. The vertical dotted lines indicate the level of the transverse images: middle image (cranial line) and right image (caudal line). (b) Transverse BASG at the level of the atlantoaxial joint demonstrating the cyst's origin from the right side of the atlantoaxial joint. (c) Transverse BASG at the caudal aspect of C2 showing the biconvex outline and ventral bilateral location of the cyst eliciting severe compression of the spinal cord.

inflammatory conditions (meningitis), or other conditions affecting soft tissues of the high cervical region.

Results of a blood and urine analysis were within normal limits.

Anesthesia and diagnostic imaging were performed as previously described in a prior study [8].

MRI revealed a well-circumscribed extradural space-occupying lesion extending from the area of the right atlantoaxial joint to the floor of the vertebral canal of the $\mathrm{C} 2$ body. The mass had a bilobed appearance and biconvex form at the $\mathrm{C} 2$ level, with rounded cranial and caudal borders, and compressed the spinal cord ventrally from both sides. The lesion was hypointense on T1-weighted images and markedly hyperintense on $\mathrm{T} 2$-weighted images. This signal suppressed in the FLAIR sequence, and an incomplete rim of contrast enhancement was present (Figure 1).

On CT, the cyst could not be visualized. An increased distance between the dens axis and the atlas, as well as an increased distance between the lamina dorsalis of the atlas and the axis were noted. Cranially to the dens axis, a small mineral dense fragment was present (Figure 2). The MRI findings, characterized by a fluid-filled lesion (with contents hypointense in T1-weighted and hyperintense in T2-weighted images) with postcontrast rim enhancement, suggested an articular cyst.

Differential diagnoses for such cystic lesions include arachnoid cysts, discal cysts, and dermoid sinus cysts, although intra- or extradural tumors and abscesses may need to be ruled out.

2.3. Surgery. A ventral midline incision and a routine approach were made at the ventral aspects of $\mathrm{C} 1$ to $\mathrm{C} 3$ [8]. A self-maintaining Gelpi retractor distracting the occipital bone and C3 allowed realignment of C1-C2. After identification of the ventral part of the atlantoaxial joint, the capsule was excised with a number 11 scalpel blade. A relatively large amount of clear synovial fluid flowed out of the joint capsule, and the remainder was aspirated. Analysis of the

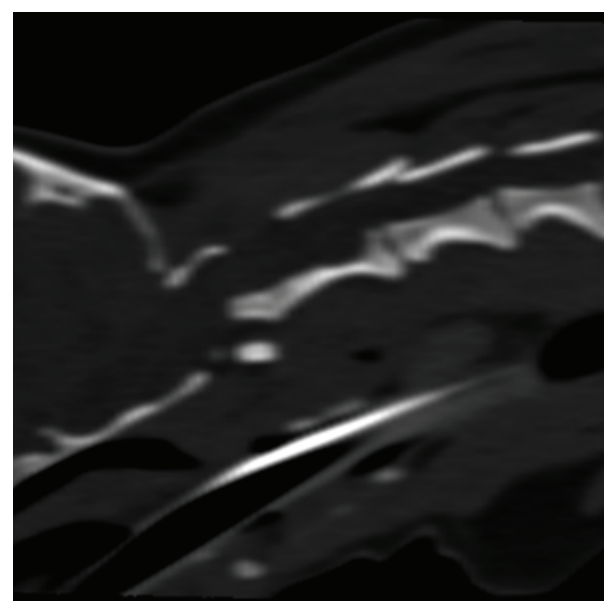

FIGURE 2: Sagittally reconstructed CT image showing the atlantoaxial subluxation and small mineral fragment cranial to the dens axis.

cyst fluid was not performed. Slight overdistraction exerted by the Gelpi retractor allowed good atlantoaxial opening and visualization of the dens, the articular cartilage and the dorsal cyst wall. The cyst wall was grasped with ophthalmic forceps and fenestration of the cyst was performed. The excised tissue sample was sent for histopathologic examination. The cartilage of the atlantoaxial joint was removed, cancellous bone was placed within the joint and the joint was stabilized with a $1.5 \mathrm{~mm}$ locking plate [8].

Postoperative care and followup have also been described in a previous study [8].

The dog was ambulatory tetraparetic the day after surgery. Physiotherapy, consisting in massages and coordination training, was begun on the first day after surgery. On the third day after surgery, locomotor deficits had disappeared, and the patient was discharged.

The dog was still clinically normal two years after surgery at which time a control CT was performed (Figure 3). 


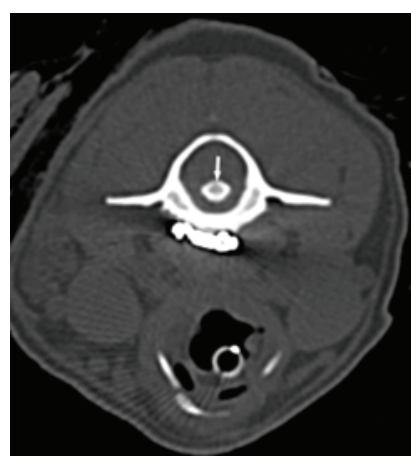

(a)

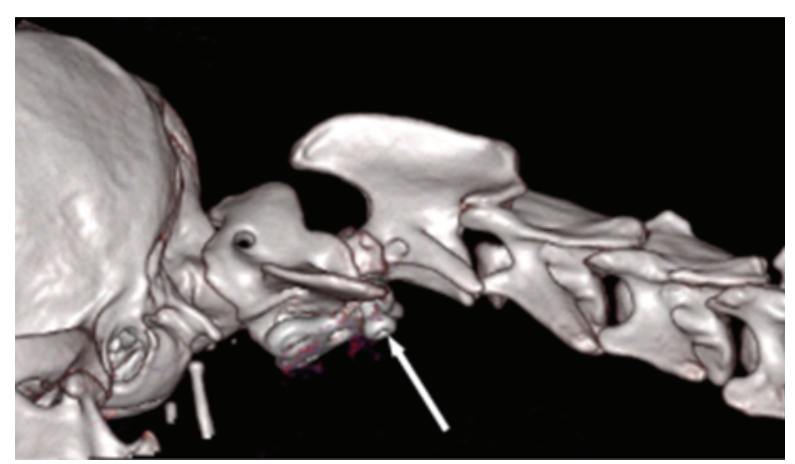

(b)

Figure 3: (a) Control CT scan after 2 years. The cyst appears not to have recurred; only a slight ventral cord compression elicited by the apex of the dens is visible (arrow). (b) Three-dimensional CT reconstruction showing the satisfactory positioning of the atlas and axis, and the loosening of the axial screws (arrow).

Despite loosening of the axial screws, atlantoaxial alignment had been preserved. It was not possible at this time to detect if synovial cyst had recurred, even after careful comparison between the control and the preoperative CT.

2.4. Histopathology. Tissue from the cyst wall was fixed in $10 \%$ buffered formalin, embedded in paraffin, sectioned at $5 \mu \mathrm{m}$, and stained with hematoxylin and eosin and Goldner's trichrome. The cyst wall was comprised of a thick layer of fibrous capsule with an inner layer of hypertrophic synoviocytes with apparent sloughing of cells into the cyst's lumen. Histopathological diagnosis was an intraspinal synovial cyst.

\section{Discussion}

Spinal articular cysts are a rare pathology in dogs and to the authors' knowledge have not been reported at the atlantoaxial joint until now. Approximately 30 cases have been described in the human literature $[9,10]$. In people, cysts associated with the atlantoaxial joint are an infrequent cause of cervicomedullary compression [7].

The atlantoaxial articulation is a true synovial joint and is responsible for a large proportion of normal cervical mobility. The etiology of articular cysts is unclear, but they are assumed to be degenerative because minor chronic damage to articular surfaces produces a reactive proliferation of synovium or fibrocartilage that includes loculated collections of mucinous fluid [11]. Cysts may contain variable amounts of proteins, blood, or both. Pathological reports traditionally divide these cysts into two types: synovial cysts, which have an epithelial lining, and ganglion cysts, which do not. Synovial cysts are the result of synovial outpouchings through weakened capsular tissue [5]. Ganglion cysts arise from mucinous degeneration of the periarticular connective tissue [5]. Both are thought to develop secondary to degenerative joint disease. Degenerative osteoarthritic changes are frequently found at the level of the cyst, however the cyst and synovial space may be discontinuous [5]. There appears to be no significant clinical difference between these two cyst types. Although the term "juxtafacet cyst" has been used to include both entities, the more general "spinal degenerative articular cyst" has been suggested because the atlantoaxial articulation is not a true facet [11].

In humans, synovial cysts are commonly located at the L4-L5 level of the lumbar spine. This segment experiences the greatest range of movement and is the most common site for degenerative disease. These findings may indicate that excessive stress to the facet joint is important in cyst generation [9]. We presume that a similar mechanism may be responsible for atlantoaxial cyst formation, namely, excessive stress to the atlantoaxial joint from repetitive chronic trauma associated with instability, which would lead to proliferation of synovium or fibrocartilage, and subsequent cyst formation. The same hypothesis has been formulated for atlantoaxial degenerative articular cysts in humans [9].

In the majority of cases, synovial cysts can be accurately differentiated from other lesions by their neuroanatomical location and specific MRI signal characteristics and contrast enhancement pattern. The classic appearance of a spinal synovial cyst is that of a well-defined, sharply marginated lesion adjacent to a degenerated facet joint, presenting signal intensities similar to cerebrospinal fluid (iso/hypointense on T1 and hyperintense on T2 weighted images) with peripheral rim enhancement after intravenous gadolinium administration [12]. Cysts may be isointense to CSF on T1W and $\mathrm{T} 2 \mathrm{~W}$ images if the contents are clear synovial fluid, or there may be high signal intensity on T1W images and T2W images if there is gelatinous or mucinous cyst material [5]. A communication between the synovial cyst and the facet joint can also be identified in some cases [2].

Nonetheless, a final diagnosis of atlantoaxial synovial cyst relies on histologic confirmation. Histopathologically, the cyst walls consist of fibrous or cartilaginous tissue and are usually lined with synovial epithelium, in part or along their entire surface, although this lining may be lacking in some cases [13]. Histological analysis suggests that inflammation does not play an important role in cyst formation.

In humans, various surgical approaches have proved to be equally effective in achieving adequate decompression, and in cases where cysts were incompletely removed, no 
ensuing complications or recurrences have been encountered $[7,11,14]$. Conservative treatment has also been described $[13,15,16]$. Furthermore, the possibility of a spontaneous regression of synovial cysts is a well-known but poorly understood phenomenon, at least in the lumbar spine [15]. One possible explanation would be a rupture of the cyst wall with leakage of the liquid content. A second and maybe more realistic possibility would be the reversal of the abnormal and prolonged microstresses applied to the joint that represent the primum movens of the cyst development [15]. In our case, the absence of clinical recurrence of the atlantoaxial degenerative articular cyst after ventral C1-C2 fusion may fit with this theory. In this paper it is surmised that the primary cause of progressive cystic degeneration was atlantoaxial instability, and stabilization of the joint may have allowed regression of the cyst. Although surgical management led to a good neurological outcome and no clinical cyst recurrence, the natural history of this atlantoaxial degenerative articular cyst remains unknown.

\section{References}

[1] C. S. H. Sale and K. C. Smith, "Extradural spinal juxtafacet (synovial) cysts in three dogs: case report," Journal of Small Animal Practice, vol. 48, no. 2, pp. 116-119, 2007.

[2] R. E. Levitski, A. E. Chauvet, and D. Lipsitz, "Cervical myelopathy associated with extradural synovial cysts in 4 dogs," Journal of Veterinary Internal Medicine, vol. 13, no. 3, pp. 181186, 1999.

[3] B. Perez, E. Rollan, F. Ramiro, and M. Pumarola, "Intraspinal synovial cyst in a dog," Journal of the American Animal Hospital Association, vol. 36, no. 3, pp. 235-238, 2000.

[4] P. J. Dickinson, B. K. Sturges, W. L. Berry, K. M. Vernau, P. D. Koblik, and R. A. LeCouteur, "Extradural spinal synovial cysts in nine dogs," Journal of Small Animal Practice, vol. 42, no. 10, pp. 502-509, 2001.

[5] A. A. Webb, J. W. Pharr, L. J. Lew, and K. A. Tryon, "MR imaging findings in a dog with lumbar ganglion cysts," Veterinary Radiology and Ultrasound, vol. 42, no. 1, pp. 9-13, 2001.

[6] F. Forterre, S. Kaiser, M. Garner et al., "Synovial cysts associated with cauda equina syndrome in two dogs," Veterinary Surgery, vol. 35, no. 1, pp. 30-33, 2006.

[7] M. Zorzon, M. Skrap, S. Diodato, D. Nasuelli, and B. Lucci, "Cysts of the atlantoaxial joint: excellent long-term outcome after posterolateral surgical decompression. Report of two cases," Journal of Neurosurgery, vol. 95, supplement 1, pp. 111114, 2001.

[8] M. Dickomeit, L. Alves, M. Pekarkova, D. Gorgas, and F. Forterre, "Use of a $1.5 \mathrm{~mm}$ butterfly locking plate for stabilization of atlantoaxial pathology in three toy breed dogs," Veterinary and Comparative Orthopaedics and Traumatology, vol. 24, no. 3, pp. 246-251, 2011.

[9] S. Marbacher, A. Lukes, I. Vajtai, and C. Ozdoba, "Surgical approach for synovial cyst of the atlantoaxial joint: a case report and review of the literature," Spine, vol. 34, no. 15, pp. E528-E533, 2009.

[10] J. J. Van Gompel, J. M. Morris, J. L. Kasperbauer, D. E. Graner, and W. E. Krauss, "Cystic deterioration of the C12 articulation: clinical implications and treatment outcomes," Journal of Neurosurgery, vol. 14, no. 4, pp. 437-443, 2011.
[11] B. D. Birch, A. G. Khandji, and P. C. McCormick, "Atlantoaxial degenerative articular cysts," Journal of Neurosurgery, vol. 85, no. 5, pp. 810-816, 1996.

[12] L. Mendes-Araújo, C. Rangel, R. C. Domingues, and E. L. Gasparetto, "Atlantoaxial synovial cyst causing isolated unilateral hypoglossal nerve paralysis," British Journal of Radiology, vol. 83, no. 986, pp. e35-e38, 2010.

[13] T. Sagiuchi, S. Shimizu, R. Tanaka, S. Tachibana, and K. Fujii, "Regression of an atlantoaxial degenerative articular cyst associated with subluxation during conservative treatment: case report and review of the literature," Journal of Neurosurgery, vol. 5, no. 2, pp. 161-164, 2006.

[14] S. Eustacchio, M. Trummer, F. Unger, and G. Flaschka, "Intraspinal synovial cyst at the craniocervical junction," Zentralblatt fur Neurochirurgie, vol. 64, no. 3, pp. 86-89, 2003.

[15] P. C. Cecchi, M. T. Peltz, P. Rizzo, A. Musumeci, G. Pinna, and A. Schwarz, "Conservative treatment of an atlantoaxial degenerative articular cyst: case report," Spine Journal, vol. 8, no. 4, pp. 687-690, 2008.

[16] O. Velán, A. Rabadán, L. Paganini, and L. Langhi, "Atlantoaxial joint synovial cyst: diagnosis and percutaneous treatment," CardioVascular and Interventional Radiology, vol. 31, no. 6, pp. 1219-1221, 2008. 

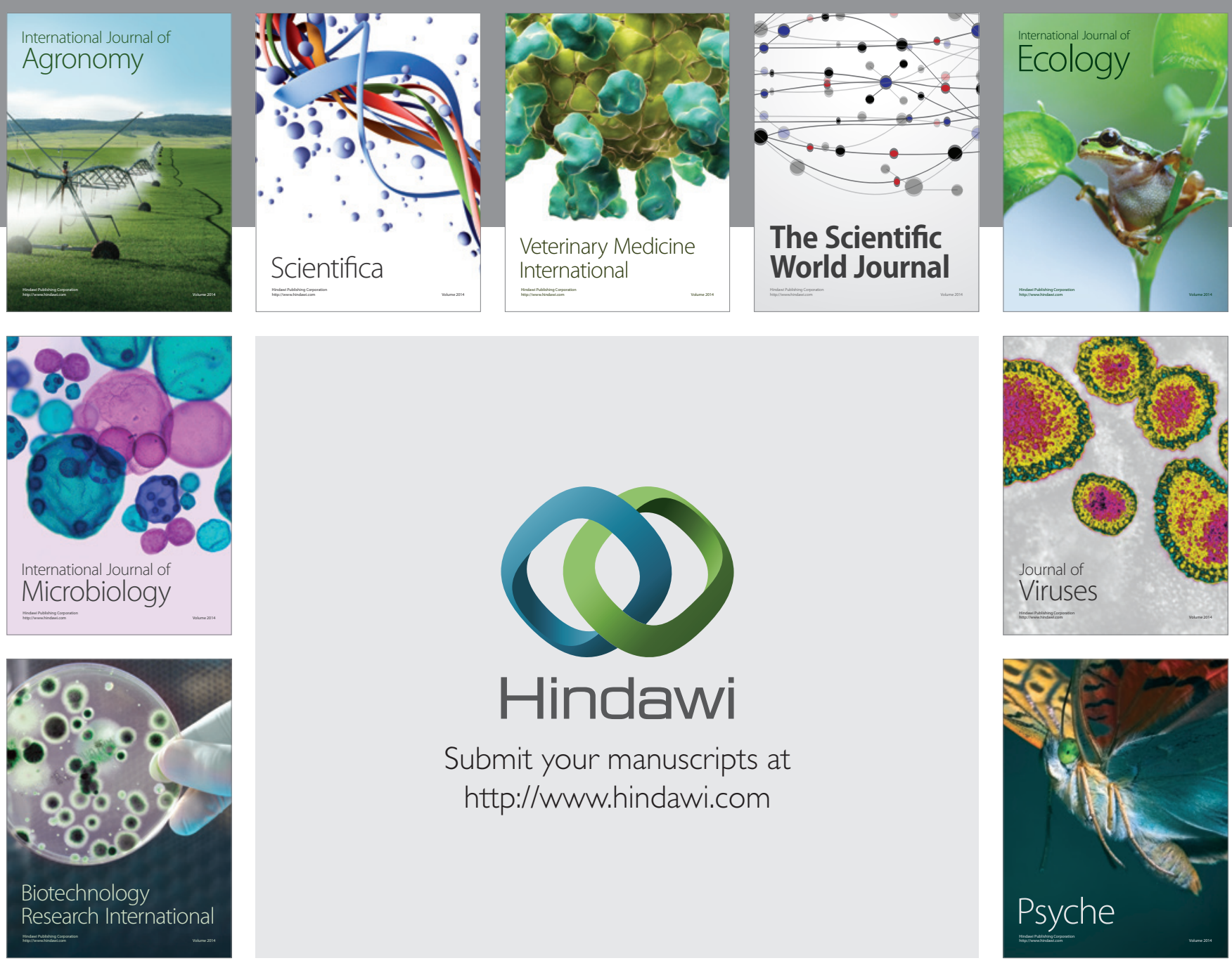

Submit your manuscripts at

http://www.hindawi.com
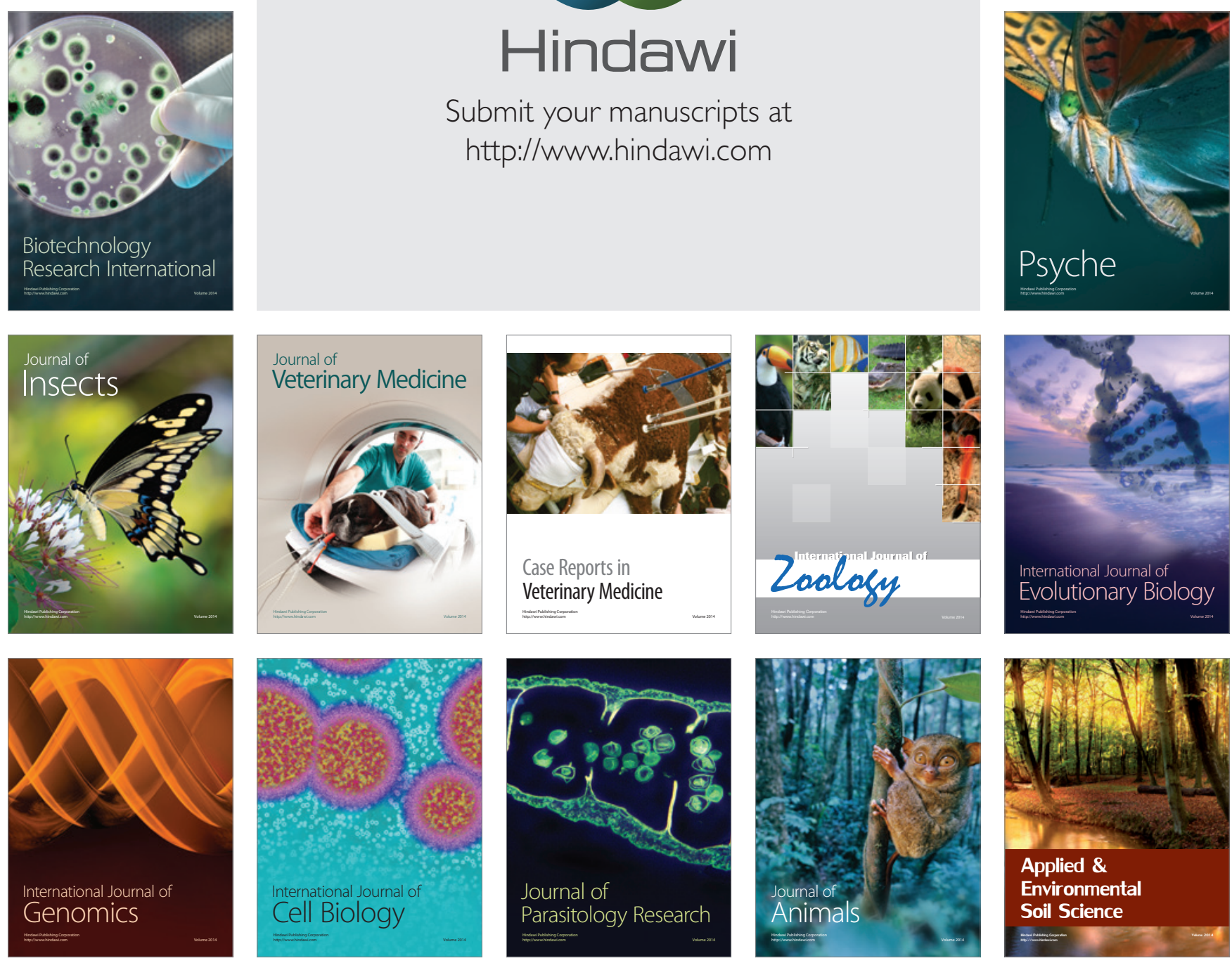